\title{
Beitrag zur Kenntnis der Frühkatatonie.
}

\author{
Von \\ Dr. Karl Pönitz, \\ zurzeit Assistenzarzt an der psychiatrischen Klinik in Halle a. S.
}

(Aus der psychiatrischen und Nervenklinik der Universität Leipzig [Direktor: Geh. Rat Prof. Dr. Flechsig].)

Mit 1 Textfigur.

(Eingegangen am 13. September 1913.)

Wiederholt ist die Vermutung ausgesprochen worden, daß wir es bei der Dementia praecox mit einer Geisteskrankheit zu tun haben, die durch eine Selbstvergiftung infolge Stoff wechselstörung bedingt ist. Man hat auf die Drüsen mit innerer Sekretion hingewiesen, auf Hoden, Ovarien, besonders auch auf Störungen in der Funktion der Schilddrüsen. K rae pelin schreibt dazu:

„Das gelegentliche Vorkommen von An- und Abschwellungen der Thyreoidea, auffallenden Hautverdickungen, Facialisphänomen, Beschleunigung oder Verlangsamung des Pulses, tetanieartigen Anfällen erinnert geradezu an die bei Schilddrüsenerkrankungen beobachteten Erscheinungen; umgekehrt bieten nach Blu ms Schilderungen die Störungen des Seelenlebens und der Bewegungen bei Hunden ohne Schilddrüse eine gewisse Ähnlichkeit mit dem Verhalten katatonischer Kranker. Lundborg hat daher die Dementia praecox in Verwandtschaft mit der Myoklonie, Myotonie und Tetanie gebracht und als ihre Grundlagen Veränderungen der Schilddrüsen und Nebenschilddrüsen angesehen."

Trotz der immer wieder ausgesprochenen Vermutungen, daß es sich bei der Dementia praecox um eine Stoffwechselstörung handelt, sind eindeutige Beweise dafür bisher nicht erbracht worden. Erst in den letzten Monaten scheint durch die Forschungsergebnisse Abderhaldens, wie sie zuerst von $\mathrm{Fauser}$ auf die Psychiatrie angewandt worden sind, die Möglichkeit gegeben zu sein, dem Ziele näherzukommen.

Es ist eine allgemein anerkannte Erfahrungstatsache, daß die überwiegende Mehrzahl der Fälle von Schizophrenie in der Zeit vom 15. bis 30. Lebensjahre manifest wird. Andererseits sind eine größere Anzahl von Fällen bekannt, die in den Kinderjahren erkranken und den ausgesprochenen Symptomenkomplex zeigen, wie wir ihn bei dər Dementia praecox kennen.

Es dürfte nun wohl die Hypothese nicht von der Hand zu weisen sein, daß bei diesen Fällen, die so auffallend früh beginnen, auch die Faktoren 
besonders ausgeprägt sind, die ätiologisch für die Erkrankung in Betracht kommen. Diese Utberlegung ist es, welche mich veranlaßte, den folgenden Fall einer kindlichen Dementia praecox, wie or in der psychiatrischen Klinik zu Leipzig zu beobachten ist, näher darauf zu untersuchen und ihn mitzuteilen.

Ein weiterer, in gewisser Beziehung äußerer Anlaß zur Untersuchung war in dem Habitus des Kindes gegeben, das lebhaft an einen Myxödematösen erinnerte. An eine Störung der Schilddrüsenfunktion zu denken lag also nahe. Ich habe daher die Schilddrüsenfunktion zu prüfen versucht, und zwar über den Weg des vegetativen Nervensystems. Ich habe mich also im besonderen der Forschungsergebnisse der Wiener Schule (Falta, Eppinger, Heß usw.) bedient.

Ich bringe zunächst die Krankengeschichte:

Oskar W., Schulknabe, Landwirtssohn, zur Zeit der ersten Aufnahme (I6. III. 1911) 13 Jahre alt.

Anamnese, von den Eltern erhalten: Ein Vetter des Pat. soll sich in einer Nervenheilanstalt befinden. Im übrigen ist von Geisteskrankheiten oder Trunksucht in der Familie nichts bekannt. Die 6 Geschwister des Pat. sind gesund, keins ist im GröBenwachstum zurückgeblieben, keins ähnelt im Habitus dem Pat. Es ist hervorzuheben, daß Oskar 10 Jahre nach dem letzten Kinde als "schwere Geburt" zur Welt gekommen ist. Er lernte mit 2 Jahren laufen, mit 3 Jahren sprechen. Er war von jeher etwas ,nervenkrank und zapplig“". Hatte schon als Kind „Anfälle“, d. h. er war sehr erregbar, sehr unrein. Keine Zuckungen! In der Schule kam er gut mit fort, nur im Rechnen war er schlecht. Dagegen hat er sehr gut auswendig gelernt. Im August 1909 soll er plötzlich heiser geworden sein, die Heiserkeit hat bis jetzt unverändert angehalten. Seitdem soll er schüchtern und komisch sein. Er gehe nie mit anderen Jungen, ziehe sich albern an, indem er irgendwelche Kleidungsstücke umhängt.

Der Zustand des Pat. verschlimmerte sich all.

Fig. 1. mählich. Oskar konnte sich mit niemandem vertragen, bekam - ohne äußeren Anlaß - sehr heftige Erregungszustände, schlug um sich, schrie: Man wolle ihn ermorden, sie wären alle verrückt, er sei nicht verrückt. In den Anfällen ist Pat. gar nicht zu bändigen, nach den Anfällen ist er schlapp und matt. Die Wutanfälle treten auch nachts auf, und es hat den Anschein, als ob Pat. dabei Gestalten um sich sieht. Pat. war bereits einige Zeit in poliklinischer Behandlung.

Körperlicher Befund: Gut genährter, untersetzter Junge. Infantiler 
Habitus. Das Gesicht läßt auf kein bestimmtes Alter schließen. Finsterer Gesichtsausdruck, Stirn in Falten gelegt. (Fig. 1.)

Größe 131. Gewicht: $61 \mathrm{~kg}$.

Schädel o. B., nur Nasenwurzel eingesunken.

Halsumfang: 321/2. Brustumfang: 79 (mittleres Maß, Pat. ist nicht zum tiefen Aus- und Einatmen zu bringen).

Mund- und Rachenorgane o. B.

Herz: Grenzen normal, Töne rein.

Puls: Gut gefüllt und gespannt, regulär, äqual.

Lunge 0 . B.

Bauchorgane und Bruchpforten o. B. vergenz.

Pupillen mittel- und gleichweit, reagieren prompt auf Lichteinfall und Kon-

Augenhintergrund und Hirnnerven o. B.

Conjunctival- und Cornealreflexe + .

Patellarreflexe + , beiderseits gleich, lebhaft.

Achillessehnenreflex + . Tricepsreflex + .

Bauchdeckenreflex + , gleich.

Keine Crines pubis. Keine Achselhaare. Haut pastös gedunsen.

Beim Druck auf die Haut bleiben keine Dellen bestehen. Sprache heiser, klanglos.

Wassermann im Blut negativ.

Im Blut: 24\% Lymphocyten, 70\% Neutrophile, 5\% Eosinophile, 1\% große Mononucleäre. Gesamtzahl der Leukocyten im ccm: ca. 7000.

Psychischer Befund bei der Aufnahme (16. März 1911): Pat. ist anfallsweise sehr unruhig und laut. Schreit, wirft sich auf den Boden, ruft nach seinen Eltern und Geschwistern, spricht laut: „Sie wollen meinen Vater totschlagen, sie wollen ihn totschlagen, sie wollen ihn totschlagen! Ich bin nicht verrückt! Mein Vater ist hier! usw." Hat den Vater angeblich vor der Türe rufen hören. Ist bei den Anfällen motorisch sehr erregt, wirft sich auf den Boden, verletzt sich dabei, sträubt sich gegen jedes Anfassen, schreit dann laut, manchmal mit voller Stimme. Dazwischen ist er wieder ruhig, apathisch. Grimassiert oft, spricht mit heiserer Stimme. Ist unrein, läßt Urin und Kot irgendwohin in die Stube. Spielt immerfort mit der Hand am Geschlechtsteil. Ist bisweilen stundenweise ganz ruhig.

20. März 1911. Pat. ist wesentlich ruhiger. Einförmig und stereotyp in Haltungen und Äußerungen. Halluzinationen sind nicht mehr zu bemerken. „Ich will nach Hause, ich will nach Hause, ich will nach Hause!" Fragen werden nicht beantwortet.

25. März 1911. Pat. redet unausgesetzt, stereotyp unsinnige Sätze vor sich hin:

„Ich weiß keine Sinichen!“

„Ich weiß keinen Handberg!"

„Ich habe an der Hand studiert!"

1. April 1911. Pat. wird heute auf dringenden Wunsch seines Vaters entlassen.

15. April 1911. Pat. wird wieder aufgenommen. Der Vater äußert jetzt den Wunsch, ihn länger in der Anstalt zu lassen. Er hat zu Hause keine Besserung ge. zeigt, vielmehr in steten Erregungszuständen Vater und Mutter angefallen, zerkratzt, gewürgt usw.

17. April 1911. Nachdem Pat. in den ersten Tagen ziemlich ruhig war, gerät or heute im Garten wieder in stärkere Erregung. Schreit oftmals laut auf. Onaniert nicht nur, wie bisher, im Zimmer, sondern auch im Garten. Ist widerspenstig.

25. April 1911. Es wechseln stets Perioden von Ruhe, Zugänglichkeit, ja 
einer gewissen Freundlichkeit mit solchen ab, in denen, stets in plötzlicher Weise einsetzend, geschrien oder ein Satz in stereotyper Weise wiederholt wird, besonders häufig: „Ich will nach Hause, ich will nach Hause, ich will nach Hause!" Nach einer solchen Erregung äußerte Pat. einmal: „Ich nehme mir's Leben!" Nachts oft unruhig, hämmert dann mit den Fäusten an den Bettrand. Hat vor kurzem grundlos einen Pat. angefallen und gewürgt. Rennt häufig mit dem Kopfe an die Wand oder stürzt sich kopfüber aus dem Bett.

27. April 1911. Bei einem Sturze aus dem Bett hat sich Pat. eine starke Beule an der Stirn zugezogen, von der aus sich Sugillationen bis in die Augenlider beider Augen erstrecken. Sonstige Zeichen einer Basisfraktur fehlen. Auffälligerweise wird die Verletzungsstelle als nicht schmerzhaft bezeichnet. Heute wieder stärkerer Erregungszustand. Pat. beruhigt sich kaum, beißt dauernd um sich, versucht auch, sich in die Hände zu beißen.

3. Mai 1911. Stark negativistisch, zeitweise unruhig und widerspenstig. Halluziniert anscheinend zeitweise sehr stark, spricht dann von der Magd zu Hause und dem Nachbar. Zu zusammenhängenden Angaben ist er nicht zu bewegen. Dabei ist er örtlich vollkommen orientiert, kennt den Arzt als solchen.

10. Mai 1911. Beim Besuche des Vaters meist nach kurzer Zeit sehr aggressiv, versucht zu beißen und zu kratzen. Spricht, in stereotyper Weise wiederholend: „Unser Pferd hat keinen Verstand.“ Spricht wiederholt laut vor sich hin: „Wir haben 4 Pferde."

15. Mai 1911. Manchmal sehr aggressiv und negativistisch. Nachts meist ruhiger Schlaf.

30. Mai 1911. Steht in gebundener Haltung da, läßt sich nicht anfassen, verweigert jede Auskunft, schimpft: „Halts Maul!“ und wiederholt nur einzelne Worte oftmals hintereinander in stereotyper Weise.

15. Juni 1911. Im allgemeinen ruhiger, doch immer noch stark negativistisch.

2. Juli 1911. Meist läppisch euphorische Stimmung, lacht sinnlos auf. Es gehe ihm sehr gut. Beim Besuche der Mutter noch leicht erregbar, hat einmal die Mutter ins Gesicht geschlagen.

26. Juli 1911. Wenig zugänglich. Läuft meist blöde lachend davon, wenn man ihn anfassen will. Lebhaftes Grimassieren! Nahrungsaufnahme schlecht, hat an Gewicht abgenommen. Hält manchmal den Mund stundenlang voll Speichel, ist aber nicht $\mathrm{zu}$ bewegen, auszuspucken.

20. August 1911. Ißt wieder besser. Psychisch kaum verändert, im ganzen vielleicht etwas stumpfer. Auch beim Besuch der Angehörigen ruhig. Von Halluzinationen zurzeit nichts zu bemerken. Keine Erregungszustände.

17. September 1911. Unverändert, steht in den Ecken umher, ist jetzt sehr gutmütig, läßt sich ruhig von anderen Patienten das Essen vom Teller fortnehmen. Zählen kann er fließend bis 100 . Zu einer eingehenden Intelligenzprüfung ist er nicht zu fixieren, läuft unter Lachen davon. Somatischer Zustand erheblich gebessert, Nahrungsaufnahme gut.

12. November 1911. Pat. sitzt stundenlang unbeweglich auf einem Fleck. Gibt nur ganz spärliche Antworten. Reagiert auf Nadelstiche nicht, es tut angeblich nicht weh. Keine Katalepsie!

25. November 1911. Etwas freier, im allgemeinen aber immer noch gedrückt und wenig zugänglich. Nahrungsaufnahme gut.

22. Dezember 1911. Ausgesprochenes Vorbeireden: Wieviel Finger? „10.“

An der r. Hand? ,,3."

An der l. Hand? ,2."

Wieviel Zehen? ,36.“

2 mal 2 ? ,9." 
2 mal $2 ?$, ,16.“

2 mal 3? "8."

Auf andere Fragen antwortet er wahllos mit „Ja" oder „Nein" oder „Das weiß ich nicht". Meist gibt er auf dieselben Fragen verschiedene Antworten.

5. Januar 1912. Andauerndes Vorbeireden, lacht dabei läppisch. Mit Spielzeug, das er zu Weihnachten bekommen hat, weiß er nichts Vernünftiges anzufangen.

2. April 1912. Finster grimassierender Gesichtsausdruck. Name und Alter werden richtig angegeben. Antwortet sehr prompt, aber ins Blaue hinein.

Wo bist du hier? "In Leipzig."

In welchem Haus? ,In der Nervenklinik."

Bist du krank? „Ja."

Was fehlt dir? "Nichts."

Bist du krank? "Nein."

Die Mimik zeigt hier plötzliche Änderung, der Gesichtsausdruck wird für Minuten freier und heiterer, wird aber bei den folgenden Fragen wieder finster.

7 mal $7 ?, 24 . “$

$7 \mathrm{mal} 13$ ? ,18."

$18-7 ?$, ,15.“

$18-7 ?, 99 . “$

$18-8 ?,, 5 . " “$

Warum siehst du so finster drein? „Ich weiß nicht, warum."

Weshalb spreche ich mit dir? "Wegen nichts."

Wie lange bist du schon hier? „,37 Jahre."

Nicht 57 Jahre? (Gleichgültig:) "Ja."

Wie alt bist du? ,14."

Wieviel Geschwister hast du? ,4 Geschwister."

Du hast doch 6! (Gleichgültig:) ,Ja, 6."

Hast du nicht 8 ? ,Ja, es sind 8."

Es sind aber doch nur 4 ? , ,Ja, es sind $4 . "$

Was willst du werden? "Schlosser."

Willst du nicht lieber Kaiser werden? (Lächelnd:) „Nein.“

Warum nicht? „Ich kann keiner werden."

Warum nicht? „Ich weiß nicht, warum."

Bist du nicht Kaiser? (Lächelnd:) „Nein, kein Kaiser.“

Lebhaftes Grimassieren, verzieht die Mundwinkel, runzelt die Stirn. Die meisten Aufforderungen werden auffallend prompt befolgt. Er zieht sich auf Aufforderungen, wenn auch sehr umständlich, aus. Passiv gegebene Stellungen werden nicht beibehalten. Beim Ausziehen beginnt er damit, sich die Hose auszuziehen. Beim Anziehen zieht er das Hemd nicht an, zieht Weste und Rock über die bloße Brust.

Warum hast du das Hemd nicht angezogen? Lacht läppisch.

20. Mai 1912. In seinem Verhalten wechselnd, bald still und finster, bald von einer läppisch-frechen Vergnügtheit. Lacht laut und viel. Schlägt grundlos $\mathrm{Pa}$ tienten. Bezeichnet Gegenstände richtig, hantiert auch richtig damit.

(Pfeife.) Referent: Ist das ein Fisch? „Ja, das ist ein Fisch." Das ist doch ein Vogel! „Eine Pfeife ist es." Schere."

(Schere.) Referent: Ist das ein Messer? „Das ist kein Messer, das ist eine

(Ball.) Referent: Ist das ein Haus? „Ja, das ist ein Haus." Das ist doch kein Haus, das ist ein Fisch! „O ja, Fisch." (Lacht dabei.) Was ist das also? "Ein Fisch." (Spielt mit dem Ball.) 
(Streichholzschachtel.) Referent: Was ist das? „Streichholzschachtel.“" Das ist doch ein Käfig! "Jawohl, das ist ein Käfig."

Wo warst du gestern? "Auf der Messe!"

Was hast du da gemacht? „Ich habe dort mit einem Manne gearbeitet, ich weiß nicht, wie er heißt."

Du warst doch den ganzen Tag hier? „Nein, ich war nicht hier."

Wann bist du denn fortgegangen? "Gestern."

Wie lange warst du fort? "55 Tage."

Wo warst du so lange? „Bei einer Frau, die die W. heißt."

Wie heißt der Kaiser? (Lachend:) „Er heißt Oskar, wie ich.“

Februar 1913. Ist im ganzen unverändert. Eigenartig sicheres, flegelhaftes Auftreten. Er hat dabei den Bewegungstyp eines behäbigen Bauern. Es besteht ein Kontrast zwischen dem Fehlen des Bartes und einer gravitätisch grimassierenden Mimik.

Pat. ist meist ruhig, redet in der oben geschilderten Weise vorbei, onaniert viel. Dazwischen jedoch heftige Erregungszustände, in denen er offenbar halluziniert. Spricht von einem Manne, der gekommen sei und von Blut. Singt dann auch mit heiserer Stimme, tanzt, schlägt auf andere Patienten los; da er dann sehr hastig spricht, ist nur ein kleiner Teil des Gesprochenen verständlich. Spricht dann völlig zusammenhanglos: „Ich bin nicht immer ganz richtig im Kopfe .... weil das Blut zu Kopfe steigt..... was soll das arme Kind immer hierin, das geht zu Kopf . . . hellrot, hellgrün . . . ist nicht recht . . . hat ihn weggeschuppt . . . es muß alles drauf ... ich kann nichts dafür, wenn ich 24 Stunden gebadet werde .... abends kam ein Schuß und ich mußte aufstehen." (Ein Schuß?) ,Ja, ein Schuß im Kopfe." Pat. spricht das alles sehr lebhaft gestikulierend, grimassierend, mit erregter Stimme, die zeitweise überschnappt. Pat. muß wegen seiner Erregungszustände wiederholt ins Dauerbad gebracht werden.

8. März 1913. Pat. liegt ruhig im Bett. Finstere Mimik. (Warum ?) „Ich weiß nicht, warum." Er bezeichnet Abbildungen und Gegenstände prompt.

Wie lange bist du hier? „Nicht lange, 4 Jahre.“

Du bist doch seit gestern da? ,Ja."

Was willst du werden? ,Sattler."

Was mußt du da machen? "Sattel machen."

Du mußt doch in die Lehre gehen! „Nein."

Kannst du gut lesen? „Sehr gut." Pat. liest prompt die Aufschriften eines Bilderbuches. Beim Ansehen des Bilderbuchs, in dem er korrekt blättert, lacht er plötzlich. (Warum?) „Ich muß lachen wegen der Geschichte." (Wegen welcher?) Zeigt auf ein Bild, äußert sich aber nicht näher. Nachdem er das Buch nach hinten durchgeblättert hat und bei einigen Bildern länger verweilte, blättert er wieder nach vorn. Bezeichnet die Bilder richtig, wenn ihm jedoch etwas anderes genannt wird, sagt er das nach.

Gefällt dir das Buch? „Ja.“

Möchtest du es haben? "Nein."

Warum nicht? „Ich weiß nicht, warum.“

Bist du schon mal mit so einem Ding gefahren? (Referent zeigt auf den Zug.) ,Ja."

Wohin denn? „Nach Eilenburg.“

Als Referent im Scherz sagt: Oskar, du siehst dir das Buch doch ganz falsch an und es ihm verkehrt gibt, lächelt Pat. und dreht es wieder.um. Spontan spricht er während allen diesen Untersuchungen nicht, blättert, während Referent schreibt, im Buche und wirft nur hin und wieder einen Blick auf ihn. Referent zeigt auf ein Bild, wo Blut aus dem Finger eines Mädchens tropft. Was ist das? „Blut.“ 
Wie kommt das dahin? "Geschnitten."

Wie alt bist du? ,25 Jahre.“

August 1913. Nach Mitteilungen aus der Klinik sind die Erregungszustände in der letzten Zeit außerordentlich heftig geworden. Pat. schreit stundenlang laut und schlägt oft wütend um sich, so daß es nicht mehr möglich ist, ihn auf einer ruhigeren Abteilung zu lassen. Eine Veränderung des Gesamtbildes ist gegen früher nicht eingetreten.

Fassen wir nun die Symptome zusammen, die der Patient bietet, so erhalten wir das einwandfreie Bild einer Schizophrenie. Wir sehen die auffälligen Störungen im Gemütsleben des Kranken: Bald steht der Junge teilnahmlos, mit apathischem Gesichtsausdruck in einer Ecke, bald läuft er singend in läppischer Euphorie im Zimmer umher und bietet unmotivierte Zornausbrüche, wobei er auch seine nächsten Verwandten mit seinen Attacken nicht verschont. Dabei ist er im höchsten Grade ungeniert in seinem Benehmen, er onaniert - wie man es so häufig bei diesen Kranken findet - in der schamlosesten Weise vor Arzt, Wärtern und Patienten. Wir sehen ferner ausgeprochene Willensstörungen: Das unsinnige Schlagen und Trommeln am Bett, das einförmige, stereotype Wiederholen ein und desselben Satzes, den bisweilen auftretenden Negativismus mit ausgesprochenem Vorbeireden. Dazu kommen noch das fast unausgesetzte Grimassieren, die - nach Krae pelin nicht seltene - vermehrte Speichelsekretion, das gleichgültige Erdulden von Nadelstichen und schließlich die mit Sicherheit anzunehmenden Sinnestäuschungen.

Wir haben also das Krankheitsbild vor uns, welches Kraepelin als ,Störung des inneren Zusammenhangs der geistigen Persönlichkeit mit vorwiegender Schädigung des Handelns und Willens" bezeichnet, die Dementia praecox oder Schizophrenie.

Wie schon in der Einleitung angedeutet, finden wir nun neben diesem psychischen Krankheitsbilde einige somatische Momente, die an eine Schilddrüsenstörung denken lassen. Es sind dies zunächst der offenbar etwas pastöse Habitus des Kindes, ferner das auffällige Zurückbleiben im Größenwachstum (die Größe des Patienten betrug bei der Aufnahme $131 \mathrm{~cm}$, während sie nach den Normalzahlen von Vierordt $142,3 \mathrm{~cm}$ hätte betragen müssen), dazu das Fehlen der Scham- und Achselhaare, das auch jetzt im 16. Lebensjahre noch zu konstatieren ist, das kleine Genitale und schließlich die auffallend heisere Sprache. Zu dem letzten Punkte sei gleich hier bemerkt, daß eine spezialistische Untersuchung des Kehlkopfes keine Aufklärung dieses Phänomens gebracht hat.

Wir kommen nun zu den Versuchen:

Zahlreiche experimentelle Untersuchungen haben mit Sicherheit ergeben, daß das Myxödem auf eine mangelhafte Funktion der Schilddrüse 
im Sinne einer Athyreosis oder Hypothyreosis zurückzuführen ist. Nun haben Kraus und Ludwig als erste gezeigt, daß bei der Basedowschen Krankheit, die ja als Hyper- resp. Dysfunktion der Thyreoidea dem Myxödem gegenüberzustellen ist, die Assimilationsgrenze für Traubenzucker herabgesetzt ist. Später hat Hirschl die schon rein spekulativ daraus zu folgernde Tatsache experimentell nachgewiesen, daß dementsprechend der Athyreoidismus die Assimilationsgrenze erhöht. Hirschl hat bis zu $500 \mathrm{~g}$ Traubenzucker verabreicht, also Mengen, die die normale Toleranzgrenze wesentlich übersteigen, und noch zuckerfreien Harn erhalten. Knöpfelmacher hat auf gleiche Weise zwei Patienten mit kongenitalem Myxödem untersucht und die Assimilation

Schema

nach Eppinger, Falta u. Rudinger:

Th.

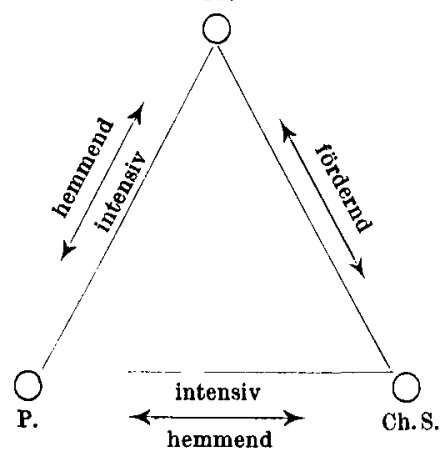

für Zucker ebenfalls abnorm hoch gefunden. Nach Zuführung von Schilddrüsensubstanz wurde diese Assimilationsgrenze herabgesetzt. Eppinger, Falta und Rudinger, die zu dem gleichen Ergebnisse in ihren Untersuchungen gelangt sind, geben für diese Tatsache folgende Erklärung: Zwischen Schilddrüse (Th.), Pankreas (P.) und chromaffinem System (Ch. S.) bestehen Wechselwirkungen, die in der nebenstehenden Figur schematisch dargestellt sind und die von den Verfassern folgendermaßen charakterisiert werden: ,1. Zwischen Th. und P., sowie zwischen $\mathrm{P}$. und Ch. S. besteht gegenseitige Hemmung, zwischen Th. und Ch. S. gegenseitige Förderung. Die Hemmungen sind intensiver als die Förderungen. 2. Ausfall bzw. Ubberfunktion der einen Drüse führt zu Uberfunktion bzw. Insuffizienz der anderen. Wir wissen nun vom Pankreas, daß es die Zuckerbildung aus dem Leberglykogen hemmt, resp. den Aufbau der Kohlehydrate in der Leber beherrscht. Umgekehrt wirkt das Nebennierensekret fördernd auf die Zuckermobilisation. Fehlt nun also die Schilddrüse oder ist - wie wir in unserem Falle vermuten - eine Hypofunktion der Thyreoidea vorhanden, so wird also einesteils der hemmende Einfluß auf das Pankreas wegfallen, d. h. das Pankreas kann mehr Kohlehydrate aufbauen als im normalen Zustande, andererseits fällt der fördernde Einfluß auf das chromaffine System weg, d. h. die Zuckermobilisation durch das Nebennierensystem wird herabgesetzt. Das Ergebnis ist demnach eine Erhöhung der Assimilationsgrenze für Zucker.

Von diesen Beobachtungen und Erwägungen wurde ausgegangen. 
Es mußte also angenommen werden, daß in unserem Falle eine abnorme Toleranz für Zucker vorhanden sein müsse. Der Patient erhielt früh nüchtern eine süße Speise, die $300 \mathrm{~g}$ Rohrzucker enthielt. Mit der Mahlzeit wurde also die normale Toleranzgrenze, die nach von Noorden für Rohrzucker zwischen $150-200 \mathrm{~g}$ liegt, bedeutend überschritten, um so mehr, als es sich um ein jugendliches Individuum handelt. Wiederholte Urinuntersuchungen, die am Versuchstage in kurzen Intervallen, am 2. und 3. Tage in längeren $Z$ wischenräumen angestellt worden sind, habən nie Zucker ergeben. Als Untersuchungsmethoden wurden einerseits das Polarisationsrohr, anderseits die Nylandersche und Trommersche Reaktion angewandt. Eine Bestimmung der Urinmenge konnte bei der Unreinlichkeit des Patienten nicht stattfinden.

Eppinger, Falta und $\mathbf{R}$ udinger haben nun weiter gezeigt, daß bei schilddrüsenlosen Tieren eine Adrenalininjektion, durch die normalerweise, wie Blum zuerst gezeigt hat, Zuckerausscheidung bewirkt wird, keine Glykosurie stattfindet; auch dann nicht, wenn gleichzeitig Zucker verfüttert wird.

Es wurde also 2 Stunden nach einer in gleicher Weise gegebenen Zuckermahlzeit 0,0007 Adrenalin subcutan injiziert und dadurch trotzdem das oben gegebene Resultat nicht beeinflußt, $d$. h. es erschien auch jetzt kein Zucker im Urin. Es ist also auf jeden Fall bei unseren Patienten eine außerordentliche Toleranz gegen Zucker festgestellt und dadurch die Wahrscheinlichkeit bedeutend erhöht werden, daß es sich um eine Hypofunktion der Schilddrüse handelt.

Neben dieser Prüfung auf Kohlehydrattoleranz wurde nun noch eine spezielle Prüfung auf Erregbarkeit des vegetativen Nervensystems unternommen. Die Drüsen mit innerer Sekretion werden, wie Biedl schreibt, einerseits von einem bestimmten Abschnitte des vegetativen Nervensystems innerviert, andererseits wirkt das innere Sekret wieder auf den Erregungszustand der entsprechenden Nerven ein. Während wir nun wissen, daß das Adrenalin das sympathische, das Pankreas das autonome System beeinflußt, wird vom Schilddrüsensekret angenommen, daß es ein polyvalentes ist, $d . h$. sowohl auf das sympathische als auf das Vagussystem wirkt, eine Ansicht, die schon Kra us (zit. nach E p pinger, Falta und Rudinger) ausgesprochen hat.

Auf diese experimentell festgestellten Tatsachen, die - neben Biedl - namentlich in den Arbeiten von Ep pinger, Falta und Rudinger, ferner Falta, Newburgh und Nobel, ferner Eppinger und $\mathrm{HeB}$, sowie Pötzl, Eppinger und $\mathrm{HeB}$, und schließlich von $\mathrm{Ba}$ u er vertreten werden, wurde gefußt, als bei unserem Patienten die Ansprechbarkeit des vegetativen Nervensystems geprüft wurde. Es mußte also erwartet werden, daß durch das mangelhafte Funktionieren der Schilddrüse nur eine geringe Wirkung derjenigen Gifte zustande 
kommt, die bekanntermaßen das vegetative System beeinflussen, d. h. des Adrenalin, des Pilocarpin und des Atropin.

Es wurde mit Adrenalin begonnen, von dem wir wissen, daß es sympathicuserregend wirkt. Eine Injektion von 0,0007 Adrenalin ergab - abgesehen von der oben erwähnten fehlenden Glykosurie - folgendes :

Keine wesentliche Änderung der Pulsfrequenz (um 96).

Keine Änderung des Blutdrucks (130).

Keine Änderung der At 2 mfrequenz (24).

Keinen Aschnerschen Reflex (d. h. keine Pulsverlangsamung bei Druck auf die Bulbi).

Pupillen bleiben mittelweit. $\mathrm{Zu}$ dem Versuch, wie auch zu den folgenden, ist zu bemerken, daß Puls, Blutdruck und zum Teil auch die Atmung graphisch aufgenommen wurden. Die Kontrolle fand während der vorangehenden Stunde und während der beiden nachfolgenden Stunden statt. In der ersten halben Stunde nach der Injektion nur mit wenigen Minuten Pause. Zu der Pulsfrequenz ist zu bemerken, daß zwei vorübergehende mäßige Steigerungen nicht zu verwerten sind, da das gleiche auch vor den Versuchen zu beobachten war.

Das Resultat des Versuchs ist also eine fehlende oder zum mindesten höchst mangelhafte Beeinflussung des sympathischen Nervensystems durch Adrenalin.

Zwei Tage später wurde 0,007 Pilocarpin, das normalerweise das autonome System erregt, injiziert. Das Ergebnis war:

Keine wesentliche Änderung der Pulsfrequenz (um 108).

Keine Änderung des Blutdrucks (130).

Leichte Änderung der Atemfrequenz (von 24 auf 20).

Pupillen bleiben weit.

Gesicht wird lebhaft gerötet.

Deutliche Salivation, die $11 / 2$ Stunde anhält.

Wir konstatieren also eine teilweise Wirkung des Pilocarpins auf das autonome System.

Wiederum zwei Tage später injizierten wir 0,0005 Atropin, also ein Gift, das das autonome System lähmt. Die Wirkung war:

Keine wesentliche Änderung der Pulsfrequenz (Puls 90-100).

Keine Änderung des Blutdrucks (130).

Keine Änderung der Atemfrequenz (um 20).

Pupillen bleiben mittelweit.

Keine sichtbare Änderung der Speichelsekretion (?).

Eine deutliche Atropinwirkung ist also nicht zu konstatieren.

Wir bekommen also durch die 3 Versuche folgendes Resultat: Eine Wirkung des Adrenalins bleibt ganz aus, ebenso ist eine solche des Atropins nicht nachweisbar. Dagegen ist eine partielle Wirkung des Pilocarpin vorhanden. Dieses Ergebnis würde unsere Annahme einer Hypo- 
funktion der Schilddrüse weiter bestärken. Denn wie schon gesagt, ist das Schilddrüsensekret ein polyvalentes, nach den Untersuchungen von Eppinger, Falta und Rudinger überwiegt aber die Wirkung auf das sympathische System. Für unseren Fall konnte man also sagen: Der Tonus des sympathischen Systems ist durch die mangelhafte Schilddrüsenfunktion herabgesetzt, Folge: mangelhafte Ansprechbarkeit auf Adrenalin. Andererseits ist der Tonus des Vagussystems weniger herabgesetzt, Folge : nur mäßige Ansprechbarkeit auf Pilocarpin. Die Atropinwirkung ist ebenfalls gering oder fehlend; Angaben über Trockenheit im Munde sind vom Patienten nicht zu erhalten, die Wirkung auf die Pupillen fehlte.

Ich bin mir natürlich wohl bewußt, daß die letzten Versuche nicht absolut beweisend für eine Hypofunktion der Schilddrüse sind. Immerhin erhöhen ihre Ergebnisse doch zusammen mit den übrigen Tatsachen die Wahrscheinlichkeit unserer Vermutung ${ }^{1}$ ).

Nachdem unsere Untersuchungen also für eine mangelhafte Funktion der Schilddrüse sprachen, lag es nahe, zu sehen, welche Wirkung unsere Versuche nach Schilddrüsenzufuhr haben würden. Es wäre zu prüfen gewesen, ob nach Verabreichung von Schilddrüsensubstanz die Resultate andere geworden wären. Es wurden also Thyreoidintabletten verabreicht, und zwar nach der Vorschrift von Falta, Newburgh und Nobel. Leider mußten wir nach kurzer Zeit damit abbrechen, da der Patient heftige Erregungszustände mit Erbrechen bekam und es mußte daher auf die Wiederholung der - nunmehr durch Thyreoidin modifizierten - Versuche verzichtet werden.

Ich resümiere: Es handelt sich um einen zurzeit 15jährigen Jungen, der im 12. Lebensjahre offensichtlich geistig erkrankt, im 13. der Klinik eingeliefert wurde. Der Symptomenkomplex der psychischen Erkrankung gleicht durchaus dem einer Dementia praecox. Also nach unserer Ansicht eine Frühform dieser Erkrankung. Kompliziert wird das Krankheitsbild durch eine Anzahl somatischer Erscheinungen, die mit ziemlicher Wahrscheinlichkeit auf eine Hypofunktion der Schilddrüse hindeuten (der leicht pastöse Habitus, Zurückbleiben im Längenwachstum, Fehlen der Schamhaare, kleines Genitale, leicht eingedrückte Nasenwurzel u. a.).

Für eine Hypofunktion sprechen ferner die auffällige Toleranz für Zucker und die mangelhafte Ansprechbarkeit des sympathischen, zum Teil auch autonomen vegetativen Nervensystems.

1) Sogar diejenigen, welche der Lehre vom „Vegetativen System“, wie sie die Wiener Schule gibt, skeptisch gegenüberstehen (Lewandowsky), halten eine Anwendung derselben auf die Störungen der Drüsen mit innerer Sekretion für berechtigt. 
Bevor zur Kasuistik der Frühkatatonie übergegangen werden soll, muß man sich folgendes klar machen:

Der Frage nach Frühformen der Dementia praecox kann auf zwei verschiedenen Wegen nähergetreten werden. Entweder sucht man die Schicksale der Schizophrenen in die Jugend zurückzuverfolgen, d. h. man verläßt sich auf die durch Angehörige gegebene Anamnesen, oder - und darum handelt es sich hier - man sucht früh beginnende Fälle selbst zu beobachten. Hierbei muß nun aber von vornherein daran festgehalten werden, daß Kinder, die in den allerersten Lebensjahren mit katatonen Symptomen erkranken, nicht mit Sicherheit zur Dementia praecox gerechnet werden können. Wenn wir in der folgenden Kasuistik bei Heller, Sante de Sanctis, Constantini und Vogt Fälle im dritten oder vierten Lebensjahre beschrieben finden, so müssen wir hier der Diagnose gegenüber skeptisch bleiben. Kraepelin definiert die Dementia praecox als ,,eine eigenartige Zerstörung des inneren Zusammenhangs der psychischen Persönlichkeit mit vorwiegender Schädigung des Willens und Gemütslebens", Ble uler hat das Charakteristische der Erkrankung in dem Worte ,Schizophrenie" ausgedrückt. Es erscheint mir nun sehr verfänglich, im dritten Lebensjahre einen Zerfall der Persönlichkeit nachzuweisen. Die typischen motorischen Symptome allein sind aber, worauf We ygandt hingewiesen hat, im frühen Kindesalter nicht so wie in den späteren Lebensjahren zu verwerten. Denn im Gegensatze zu Kraepelin, der geneigt ist, auch gewisse Formen der Idiotie als Frühformen der Dementia praecox aufzufassen, meint Weygandt, daß die zahlreichen, der Dementia praecox ähnlichen motorischen Störungen sich aus einer gewissen Entwicklungsperiode der normalen Kindheit erklären.

Für unseren Fall kommen alle diese Bedenken nicht in Betracht, da die Krankheit erst nach dem 10. Lebensjahre offensichtlich wurde. Dabei ist allerdings nicht zu leugnen, daß der Knabe schon als Kind eigenartig gewesen ist. Es handelt sich nach der Anamnese, die leider nicht genauer zu erheben war, um eigenartige degenerative Störungen, die man nicht sicher klassifizieren kann. Da die Schulleistungen des Patienten zum Teil gute waren, ist man aber kaum berechtigt, von einer Imbezillität zu sprechen, der Fall wäre demnach auch nicht der ,Pfropfhebephrenie" K rae pelins zuzurechnen.

Ich gehe nun kurz auf die Kasuistik ${ }^{1}$ ) der Frühkatatonie ein:

Schon Kahlbaum, der Begründer der Katatonielehre (zit. nach Raecke) hat darauf hingewiesen, da $B$ die Katatonie auch im Kindesalter vorkommen kann. Auch Kraepelin gibt zu, mehrere Fälle von Dementia praecox im Kindesalter beobachtet zu haben.

1) In chronologischer Reihenfolge. Eine vollständige Kasuistik zu geben ist nicht beabsichtigt. 
Bereits im Jahre 1875 hat Kelp drei Fälle von Kinderpsychosen veröffentlicht, von denen der zweite lebhaft an das Bild einer Katatonie erinnert, wenn auch der Name „Katatonie“ nicht vom Verfasser gebraucht wird.

Als „Dementia infantilis“ hat Heller eine Anzahl Erkrankungen geschildert, von denen ein Teil mit Wahrscheinlichkeit der Dementia praecox angehört. Bei einem kleinen Teil der Fälle ist meines Erachtens wie ich schon oben angedeutet habe, die Diagnose nicht mit Sicherheit zu stellen.

Auch E. Meyer (zit. nach Raecke) hat Katatonien im 12. und 14. Lebensjahre wiederholt gesehen, von Ziehen wurden Fälle bis ins 7. Lebensjahr zurückverfolgt. Ziehen will in manchen Fällen Symptome einer abnorm frühen Pubertätsentwicklung bemerkt haben.

Im Jahre 1906 hat Sante de Sanctis, nachdem er einige Jahre vorher drei ähnliche Fälle beschrieben hat, den Fall eines dreijährigen Mädchens veröffentlicht, das nach Schreck erkrankte und das Symptomenbild einer Katatonie bot. Sa nte de Sanctis weist jedoch darauf hin - und damit bestätigt er meine Bedenken bezw. der Diagnose derartiger Fälle --, daß die Diagnose nur auf Grund der Ähnlichkeit der Symptome bei diesen Frühformen der Dementia praecox gestellt wird. Er läßt es dahingestellt, ,ob für diese Kranken die gleiche Ätiologie und Pathogenese geltend gemacht werden kann, wie sie für die von Dementia praecox Befallenen zwar nicht bewiesen, aber vermutet wird" (d. h. eine Autointoxikation). Er ist der Meinung, daß es Symptomenbilder der Katatonie gibt, die von der Dementia praecox unabhängig sind. Ich schließe mich dieser Ansicht vollständig an, soweit es sich nur um ausgesprochene motorische Symptome (,,motorisch" im engeren Sinne des Wortes) handelt und soweit daneben ein Persönlichkeitszerfall nicht festzustellen ist. Dies ist, wie schon angedeutet, u. a. der Fall bei den erwähnten ganz frühzeitigen Erkrankungen und bei gewissen Formen der Idiotie. Ist jedoch ein Persönlichkeitszerfall nachweisbar, wie es bei unserem Patienten der Fall ist, so halte ich es nicht etwa für berechtigt, die Frühkatatonie ätiologisch und pathogenetisch nur deshalb von der Dementia praecox abzutrennen, weil sie vor der Pubertätszeit manifest wird ${ }^{1}$ ). In unserem Falle, daran muß festgehalten werden, handelt es sich eben um eine typische Katatonie, die - abgesehen von der Schilddrüsenstörung - nur dadurch bemerkenswert ist, daß sie in den Kinderjahren begonnen hat.

Die größte Anzahl von ,,Katatonie im Kindesalter" hat Raec ke veröffentlicht, im ganzen 10 Fälle. Von diesen Kindern, die zwischen dem

1) Von all diesen Erwägungen bleibt natürlich die Frage unberührt, ob wir. es bei dem großen Gebiet der Dementia praecox nicht überhaupt mit einer ätio. logisch verschiedenartigen Krankheitsgruppe zu tun haben. 
12. und 15. Lebensjahre standen, galten die Hälfte als bisher normal entwickelt, zum Teil sogar als gut begabt; bei den übrigen muß man sicher von einer mangelhaften Begabung, bzw. Imbezillität sprechen. Allen ist gemeinsam der , jähe Wechsel zwischen Hemmung und Erregung mit Steigerung zu Stereotypien und triebartig Bizarrem, zu impulsiven Entladungen und zu blindem Widerstreben bei Fehlen von ausgeprägter Affektanomalie und Bewußtseinstrübung “. Ein Zweifel an der Diagnose ist nach den Krankengeschichten in keinem Falle berechtigt.

In den letzten Jahren hat noch Constantini (zit. nach Vogt) zwei Fälle von „Dementia praecocissima" beschrieben, eine im 4 . und eine im 5. Lebensjahre beginnend; und kurz nach Raecke hat Vogt drei Fälle veröffentlicht, davon wird der erste ( 5 Jahre alt) als rein katatoner Typus, der zweite (9 Jahre alt) als hebephrener Typus mit katatonen Zügen und der dritte (15 Jahre alt) als paranoider Fall charakterisiert. Vogt meint, daß aus physiologischen Gründen die katatone Form im Kindesalter überwiegt und erinnert damit an die Ansichten Weygandts.

Was also den von mir mitgeteilten Fall von den übrigen unterscheidet, sind die Momente, welche auf eine Hypofunktion der Schilddrüse hinweisen. Zum mindesten fehlen in den in der Literatur mitgeteilten Fällen Angaben über ein Zurückbleiben im Wachstum und über andere somatische Symptome. Daß in unserem Falle eine Schilddrüsenstörung vorliegt, glaube ich wahrscheinlich gemacht zu haben; sicher ist jedoch eine Störung im Bereiche der Drüsen mit innerer Sekretion vorhanden. Man wird vermuten dürfen, daß diese Störungen für das frühzeitige Auftreten der schizophrenen Erkrankung nicht ohne Bedeutung sind, zumal, wie oben gesagt, auch andere Erwägungen und Untersuchungen, die an Katatonikern gemacht worden sind, dafür sprechen.

Herrn Geheimrat Flechsig danke ich für die gütige Erlaubnis zur Publizierung des Falles, Herrn Kollegen Schilder für die Unterstützung bei den Versuchen.

\section{Literaturverzeichnis.}

\section{Zur Dementia praecox im Kindesalter:}

Constantini, Due case di dementia praecocissima. Rivista di Patologia nervosa e mentale 13, H. 3. 1908. (Zitiert nach Vogt.)

Heller, Über Dementia infantilis. Zeitschr. f. jugendl. Schwachsinn 2, 17. 1909. Infeld, Beiträge zur Kenntnis der Kinderpsychosen. Jahrb. f. Psych. 22. 1902. Kahlbaum, Die Katatonie oder das Spannungsirresein. 1874.

Kelp, Psychosen im kindlichen Alter. Allgem. Zeitschr. f. Psych. 31. 1875.

Kraepelin, Einführung in die Psychiatrische Klinik. 2. Aufl. 1905. S. 355.

- Lehrbuch der Psychiatrie. 8. Aufl. 3. 1913.

Meyer, E., Die Ursachen der Geisteskrankheiten. Jena 1904.

Raecke, Katatonie im Kindesalter. Archiv f. Psych. 45. 1909. 
Sante de Sanctis, Rivista sperim. di Freniatria Vol. XXXII, Fasc. I, II. 1906. - Dementia praecocissima. Folia neurobiol. 2, 1908.

Vogt, Über Fälle von Jugendirresein im Kindesalter. Allgem. Zeitschr. f. Psych. 66. 1909.

Weygandt, Idiotie und Dementia praecox. Zeitschr. f. jugendl. Schwachsinn 1, 311. 1907.

Ziehen, Geisteskrankheiten des Kindesalters. II. Berlin 1904.

\section{Zur Störung der Schilddrüsenfunktion:}

Ba uer, Zur Funktionsprüfung des vegetativen Nervensystems. Deutsches Archiv f. klin. Med. 10\%. 1912.

Bied1, Innere Sekretion. 1. und 2. Aufl. 1910 und 1913 (ausführliches Literaturverzeichnis ).

Blum, Über Nebennierendiabetes. Deutsches Archiv f. klin. Med. 91. 1901.

Bonhoeffer, Die Psychosen im Gefolge von akuten Infektionen, Allgemeinerkrankungen und inneren Erkrankungen. Handb. d. Psych. 1912.

Eppinger und $\mathrm{He} B$, Die Vagotonie. Berlin 1910.

- Falta und Rudinger, Über die Wechselwirkungen der Drüsen mit innerer Sekretion. Zeitschr. f. klin. Med. 66. 1908.

Falta, Newburgh und Nobel, Über die Wechselwirkungen der Drüsen mit innerer Sekretion. Zeitschr. f. klin. Med. 72. 1911.

Hirschl, Beiträge zur Kenntnis des Morbus Basedowii. Jahrb. f. Psych. u. Neurol. 22. 1902.

Knöpfelmacher, Alimentäre Glykosurie und Myxödem. Wiener klin. Wochenschr. 1904, Nr. 9.

Kraus und Ludwig, Klinischer Beitrag zur alimentären Glykosurie. Wiener klin. Wochenschr. 1891, S. 855.

Lewandowsky; Stand und Aufgaben der allgemeinen Physiologie und Pathologie des sympathischen Systems. Zeitschr. f. d. ges. Neur. u. Psych. 14. 1913.

v. Noorden, Die Zuckerkrankheit. Berlin 1898.

Pötzl, Hirnschwellung. Jahrb. f. Psych. 31. 1910.

- Eppinger und HIeB, Über Funktionsprüfung des vegetativen Nervensystems bei einigen Gruppen von Psychosen. Wiener klin. Wochenschr. 1910, Nr. 51.

Wagner v. Jauregg, Myxödem und Kretinismus. Handb. d. Psych. 1912. 